Network Working Group

Request for Comments: 2628

V. Smyslov

Category: Informational

June 1999

Simple Cryptographic Program Interface (Crypto API)

Status of this Memo

This memo provides information for the Internet community. It does not specify an Internet standard of any kind. Distribution of this memo is unlimited.

Copyright Notice

Copyright (C) The Internet Society (1999). All Rights Reserved.

Abstract

This document describes a simple Application Program Interface to cryptographic functions. The main purpose of such an interface is to separate cryptographic libraries from internet applications, thus allowing an independent development of both. It can be used in various internet applications such as [IPsec], [ISAKMP], [IKE], [TLS] .

Table of Contents

1. Introduction. . . . . . . . . . . . . . . . . . . . 2

1.1. Summary . . . . . . . . . . . . . . . . . . . . . . 2

1.2. Terminology . . . . . . . . . . . . . . . . . . . . . . . . 2

1.3. Objectives of Development . . . . . . . . . . . . . . . 3

2. Cryptoplugin Structure. . . . . . . . . . . . . . . . . 3

3. Program Interface. . . . . . . . . . . . . . . . . . . . 4

3.1. Cryptoplugin Initialization Function. . . . . . . . . . . . . 4

3.1.1. Description of Cryptoplugininfo structure . . . . . . . . 6

3.1.2. Description of CryptoAlgInfo structure. . . . . . . . . . . 6

3.2. Cryptoplugin Deinitialization Function. . . . . . . . . . . . 9

3.3. Cryptographic Context Opening Function. . . . . . . . . . . 10

3.4. Cryptographic Context Reopening Function. . . . . . . . . . . 11

3.5. Cryptographic Context Closing Function. . . . . . . . . . . . 12

3.6. Key Verification Function . . . . . . . . . . . . . . . 12

3.7. Data Transformation Function. . . . . . . . . . . . . . 13

3.7.1. For CRYPTO_TYPE_ENCRYPT Algorithm Type. . . . . . . . . . 13

3.7.2. For CRYPTO_TYPE_DECRYPT Algorithm Type. . . . . . . . . . 14

3.7.3. For CRYPTO_TYPE_SIGN Algorithm Type . . . . . . . . . . . 15

3.7.4. For CRYPTO_TYPE_VERIFY Algorithm Type . . . . . . . . . . . 17

3.7.5. FOr CRYPTO_TYPE_COMPRESS Algorithm Type..... . . . 18 
3.7.6. FOr CRYPTO_TYPE_UNCOMPRESS Algorithm Type . . . . . . . . 18

3.7.7. For CRYPTO_TYPE_HASH Algorithm Type . . . . . . . . . . . . 19

3.7.8. For CRYPTO_TYPE_RANDOM Algorithm Type. . . . . . . . . . . 21

3.8. Cryptographic Context Control Function. . . . . . . . . . . . 22

4. Cryptoplugin Registration Procedure . . . . . . . . . . . . . . 23

5. Security Considerations . . . . . . . . . . . . . . . . . 23

6. References. . . . . . . . . . . . . . . . . . . . . 23

7. Author's Address . . . . . . . . . . . . . . . . . . . . . . 24

Appendix A. The interface specification as a C header file . . . 25

Full Copyright Statement . . . . . . . . . . . . . . . 30

1. Introduction

1.1. Summary

Nowadays internet applications that require cryptographic functions at the level of operating system kernel, use the method that assumes the libraries must be compiled/linked together with the module (driver) which provides product functionality. For the sake of possibility of independent development of the cryptographic modules and in order to provide a simple, effective and universal (suitable for application and as well kernel level of operating system) solution this specification offers the method to extract encrypting algorithms to the separate cryptographic modules.

This document describes simple open interface (Crypto API) to external cryptographic libraries optimized both for the application and kernel level of the operating system.

\subsection{Terminology}

Cryptoplugin

Operation system unit (driver, shared library, module) that provides cryptographic functions via well-defined (but OSspecific) interface.

Cryptolibrary

Part of cryptoplugin that provides its cryptographic functionality via Crypto API.

Wrapper

Part of cryptoplugin that provides interfaces translation between Crypto API and OS-specific interface. 
Definition of all cryptography related terms can be found in [Schneier].

1.3. Objectives of Development

The objectives of Simple CryptoAPI development are as follows:

1) To extract program implementations of encryption, one-way hash function, digital signature and random numbers generation algorithms to separate, independently developed modules.

2) To provide version independence between using encryption modules and external cryptoplugin.

3) To ensure platform independent developments of encrypting algorithm modules with portable source code.

4) To enable independent development of modules and compatibility of modules developed independently.

2. Cryptoplugin Structure

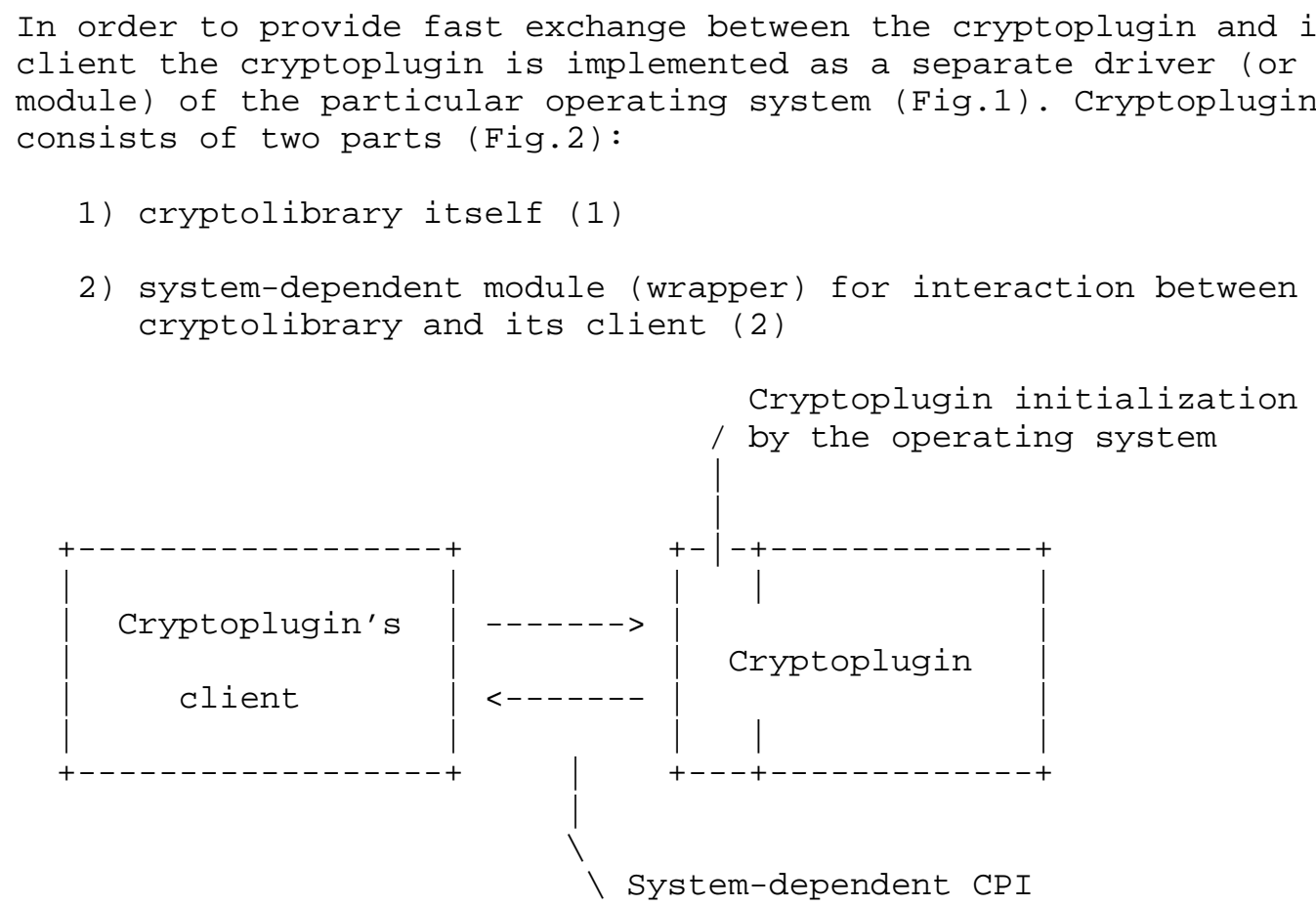

Fig. 1 Interaction between cryptoplugin and its client 


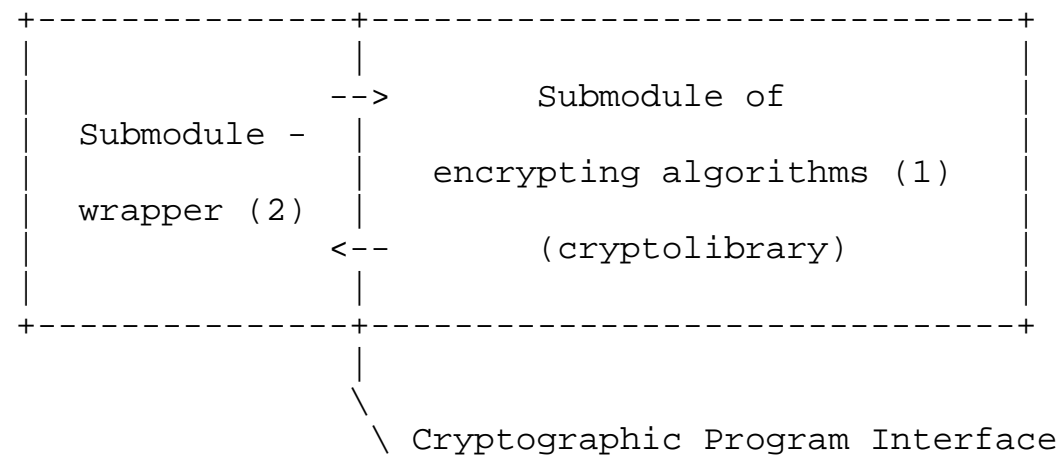

Fig. 2 Cryptoplugin structure

The system-dependent module (wrapper) is delivered by the driverclient developer in the form of source code or in the form of libraries (for example, in the form of object files) for particular operating system. The wrapper is intended for translation of system-independent application interface to the particular systemdependent interface with the cryptoplugin's client. The wrapper context does not include components specific to cryptoplugin's client functionality or to some cryptographic algorithm. The interface described in section 3 is the standard for interaction between the submodules (1) and (2).

A cryptoplugin can contain a number of different algorithms. Moreover, it can contain some different implementations of one particular algorithm.

3. Program Interface

The CPI (Cryptographic Program Interface) consists of a set of functions exported by encrypting algorithm submodule (cryptolibrary). The interface functions are described below (see also Appendix A).

\subsection{Cryptoplugin Initialization Function}

The function is intended for cryptoplugin initialization and obtaining information about algorithms contained in cryptoplugin. The function is called once before the beginning of cryptoplugin operation.

/* Cryptoplugin initialization. Returns pointer to CryptopluginInfo structure on success or NULL on fatal error. */ CryptopluginInfo *CryptopluginInit void

*param);/* Ptr to os parameters (platform-specific) */ 
Description of parameters:

param - pointer to system-dependent parameters transmitted to cryptoplugin by the operating system. Intention and format of parameters are specific to each operating system and should be described in documentation on the cryptoplugin wrapper.

The function is called at the moment of cryptoplugin initialization. If succeeded it returns the pointer to Cryptoplugininfo structure that describes the module and algorithms implemented in the cryptolibrary. If function call did not succeed, function will return NULL or appropriate error code in CryptoPluginInfo structure status field. If the initialization is partially succeeded then the cryptoplugin either returns CryptopluginInfo structure transformed so that it contains only successfully initialized algorithms or returns appropriate error code in status field of CryptoAlgInfo structures that describes the reason for the failure.

Error codes for the function:

NULL - fatal unsuccessful cryptoplugin initialization. The module is unable even to indicate the reason of failure.

The pointer to cryptoplugin description structure in the case of full or partial success. The status fields in CryptopluginInfo structure and in comprised CryptoAlgInfo structures can be set to the following values:

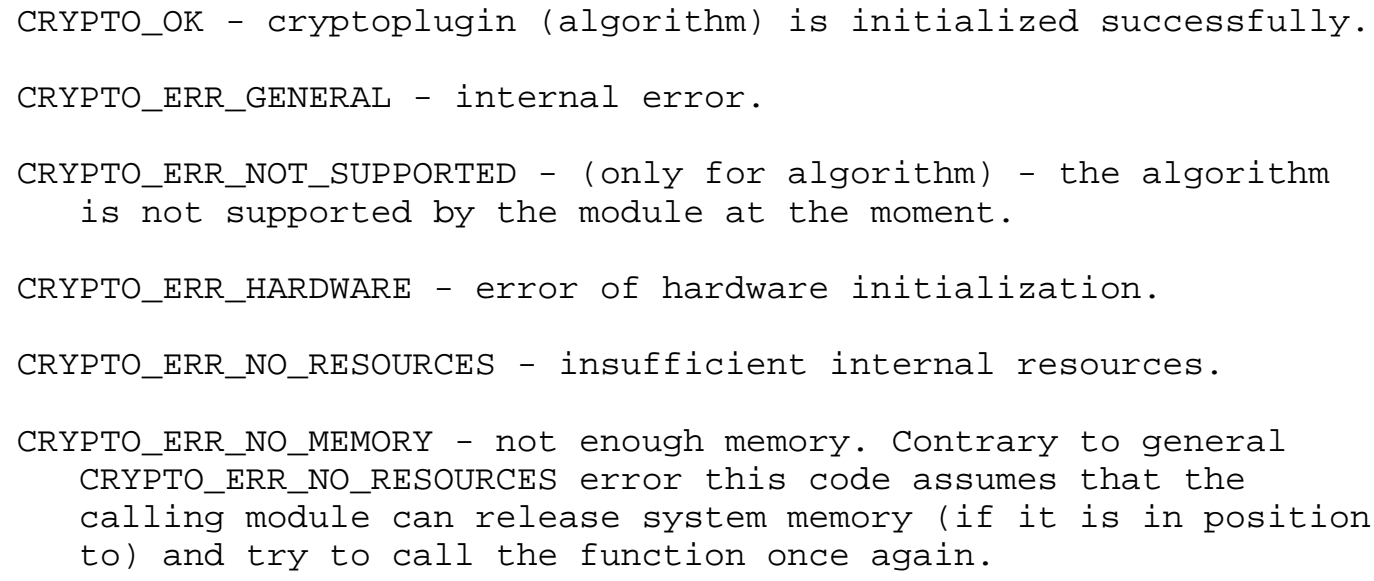




\subsubsection{Description of CryptopluginInfo structure}

The CryptopluginInfo structure consists of header of fixed size that generally describes cryptoplugin and array of CryptoAlgInfo

structures following the header. Each structure describes particular algorithm implemented in the cryptolibrary (see Appendix A)

Structure fields description:

cpi_version - CPI version (should be CRYPTO_VER (1,0)). CPI version determines both functions set and fields layout in CryptoPluginInfo/CryptoAlgInfo structures.

status - returns the error code if cryptoplugin initialization failed (otherwise should be CRYPTO_OK)

name - text cryptoplugin description (ASCII-7 characters only; all unused bytes must be set to 0 ).

version - cryptoplugin version (CRYPTO_VER(maj,min)).

flags - various flags that characterize the cryptoplugin.

number_of_algs - number of algorithms the cryptolibrary comprises of (i.e. the number of consequent CryptoAlgInfo structures).

3.1.2. Description of CryptoAlgInfo structure

Structure fields description

status - returns the error code if particular algorithm initialization failed (otherwise should be CRYPTO_OK).

id - algorithm identifier (CRYPTO_A_XXX). Values in the range of $0 . .249$ are reserved; Values in the range of 250..32767 indicate algorithms not enrolled in standard list. It should be emphasized that algorithm IDs are independent for each algorithm type. But it is considered that pairs of types CRYPTO_TYPE_ENCRYPT and CRYPTO_TYPE_DECRYPT, CRYPTO_TYPE_SIGN and CRYPTO_TYPE_VERIFY, CRYPTO_TYPE_COMPRESS and CRYPTO_TYPE_UNCOMPRESS are equivalent because they define reverse actions of the same nature. 


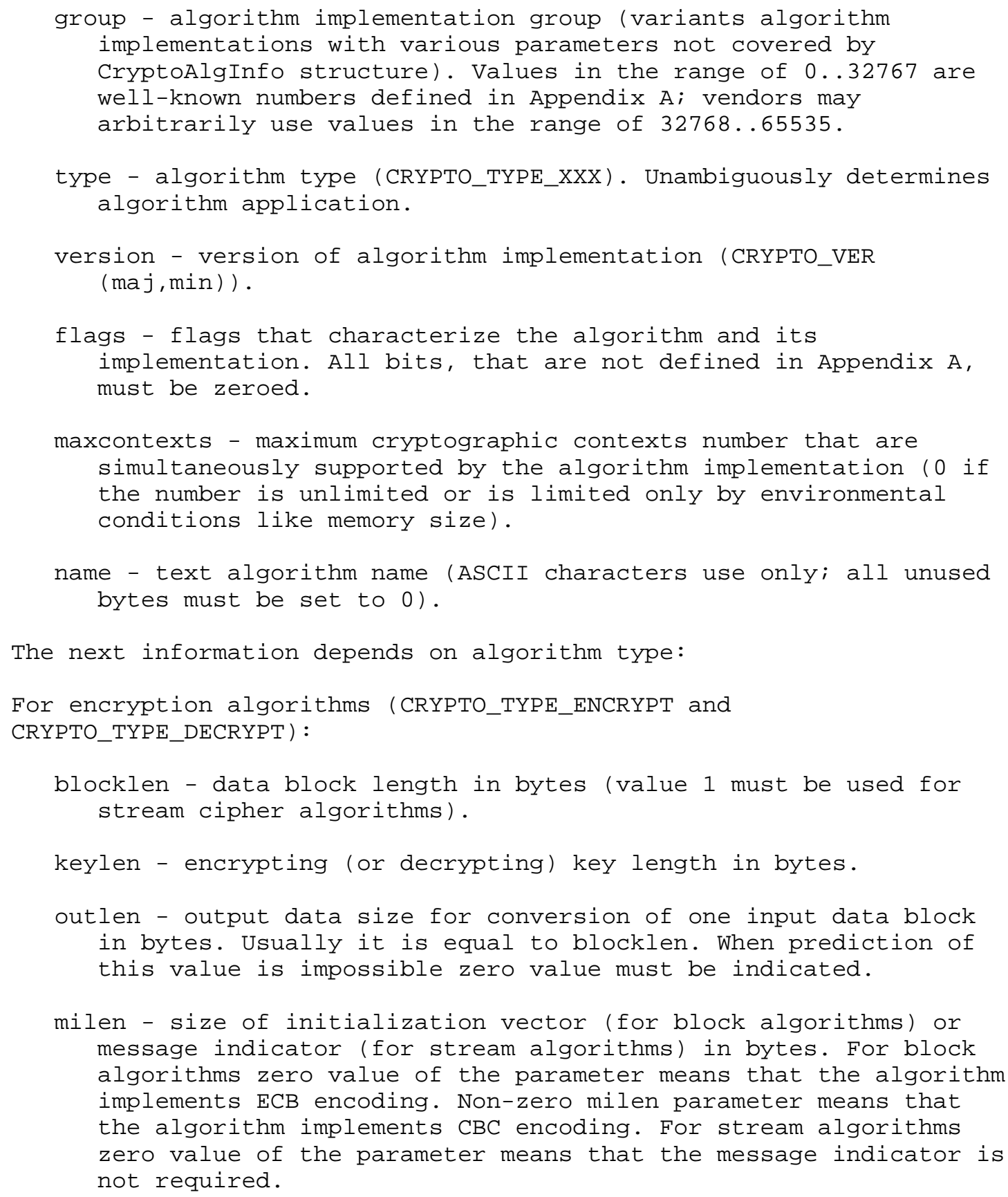




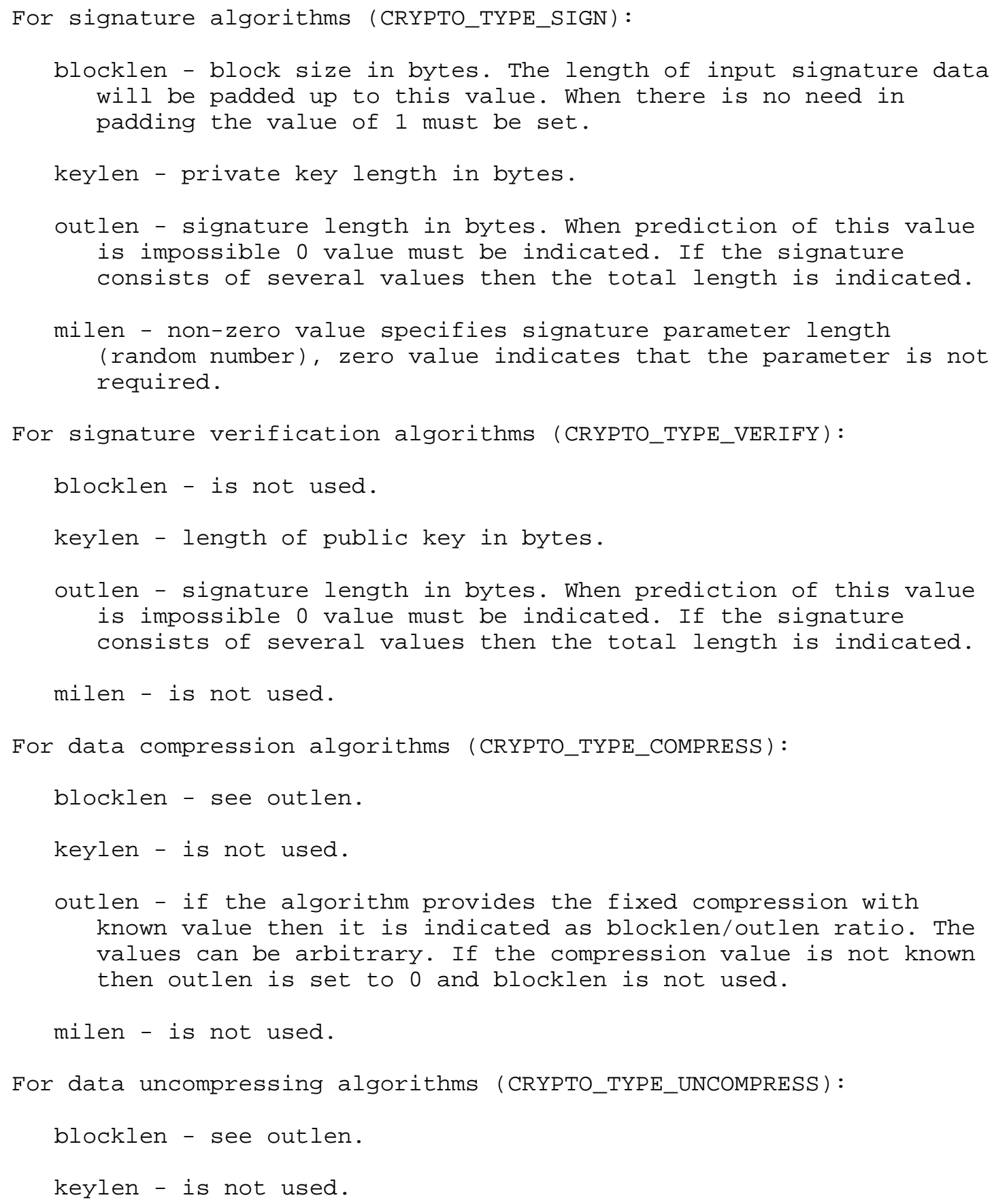




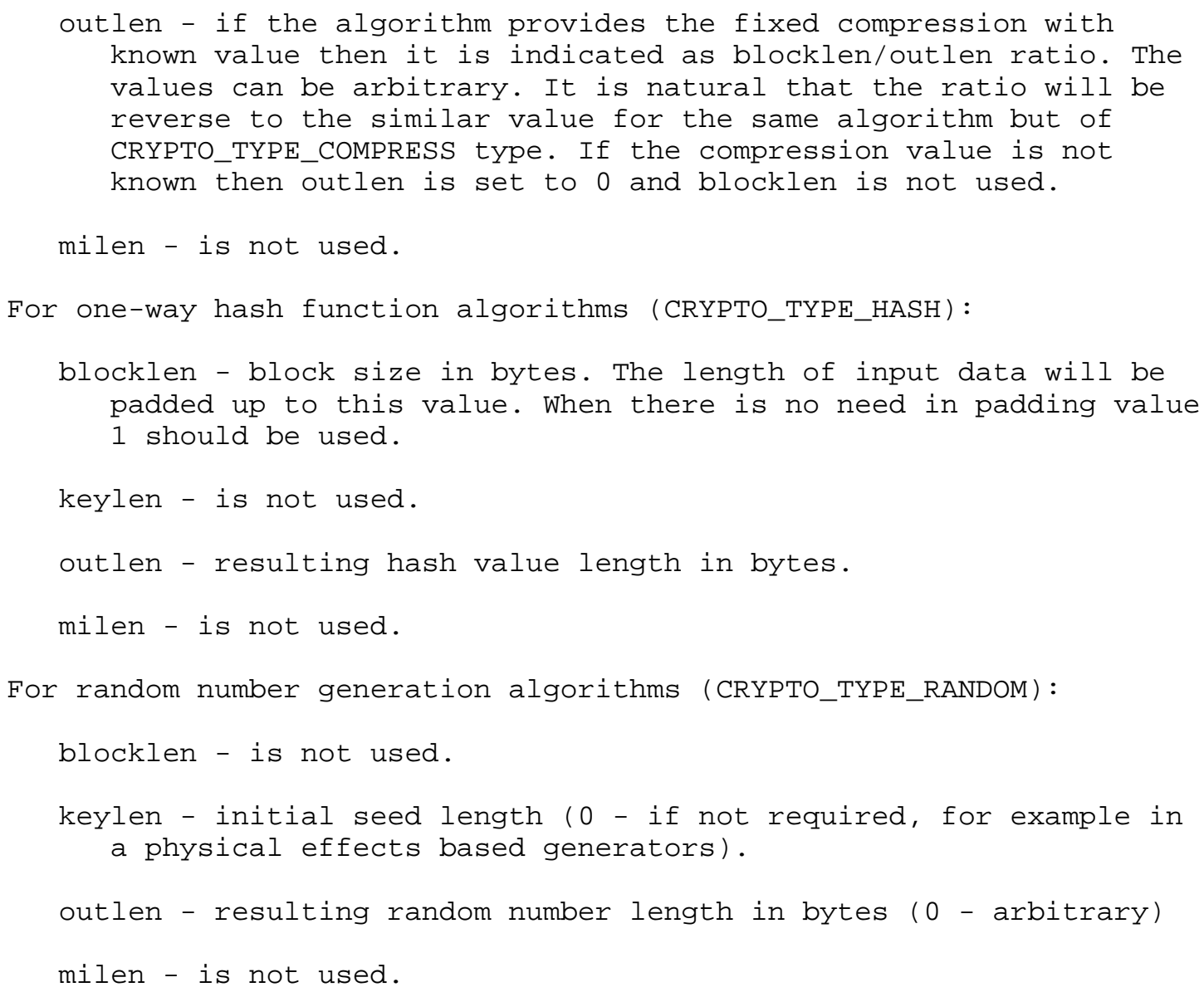

3.2. Cryptoplugin Deinitialization Function

/* Plugin deinitialization */

CRYPTO_STATUS CryptoPluginFini(void);

The function is called before the cryptoplugin operation is to be terminated. Function execution causes closing of all open cryptographic contexts, system resources deallocation and hardware deinitialization. The value returned is informational only.

Return codes for the function:

CRYPTO_OK - cryptoplugin is deinitialized successfully.

CRYPTO_ERR_GENERAL - internal error. 
CRYPTO_ERR_UNCLOSED_HANDLES - warning that there were open cryptographic contexts during cryptoplugin deinitialization. The warning is informational only. The open contexts are destroyed anyway.

3.3. Cryptographic Context Opening Function

New algorithm instance (cipher state) */ CRYPTO_STATUS Cryptoopen(

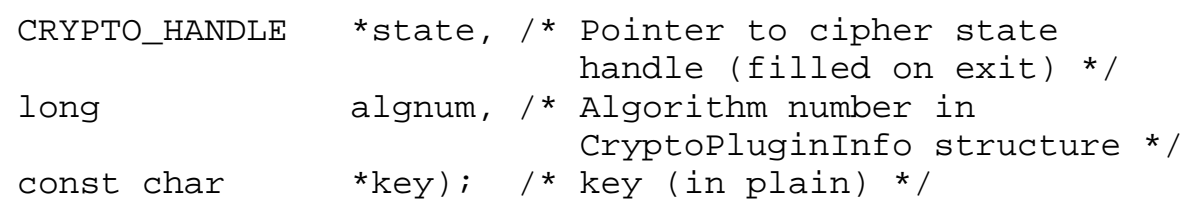

The function creates cryptographic context copy inside cryptoplugin and initializes it with the provided key. Later the handle of the context is used in calls of other algorithm functions.

Description of parameters:

state - pointer to the variable that will be set to the handle of the context created if succeeded. NULL parameter value should result in the CRYPTO_ERR_BAD_PARAMS code returned by the function.

algnum - algorithm number in the cryptoplugin. It is equal to the number of CryptoAlgInfo structure (that describes the algorithm) in CryptopluginInfo structure. The number begins with zero value. It should be taken into account that it is not an algorithm identifier but its number in the cryptoplugin.

key - pointer to the key (if it is required) or to the seed (for random number generation algorithm).

Notes.

1. Generated cryptographic context is stored inside the cryptoplugin until it will be destroyed by the CryptoAlgClose function call. The maximum number of cryptographic contexts supported by cryptoplugin can be indicated in algorithm parameters description. If maximum number of cryptographic contexts equals to zero then the cryptographic contexts number is either unlimited (for example, for stateless algorithms like random number generators and one-way hash functions) or it is limited by external factors only (like memory size). 


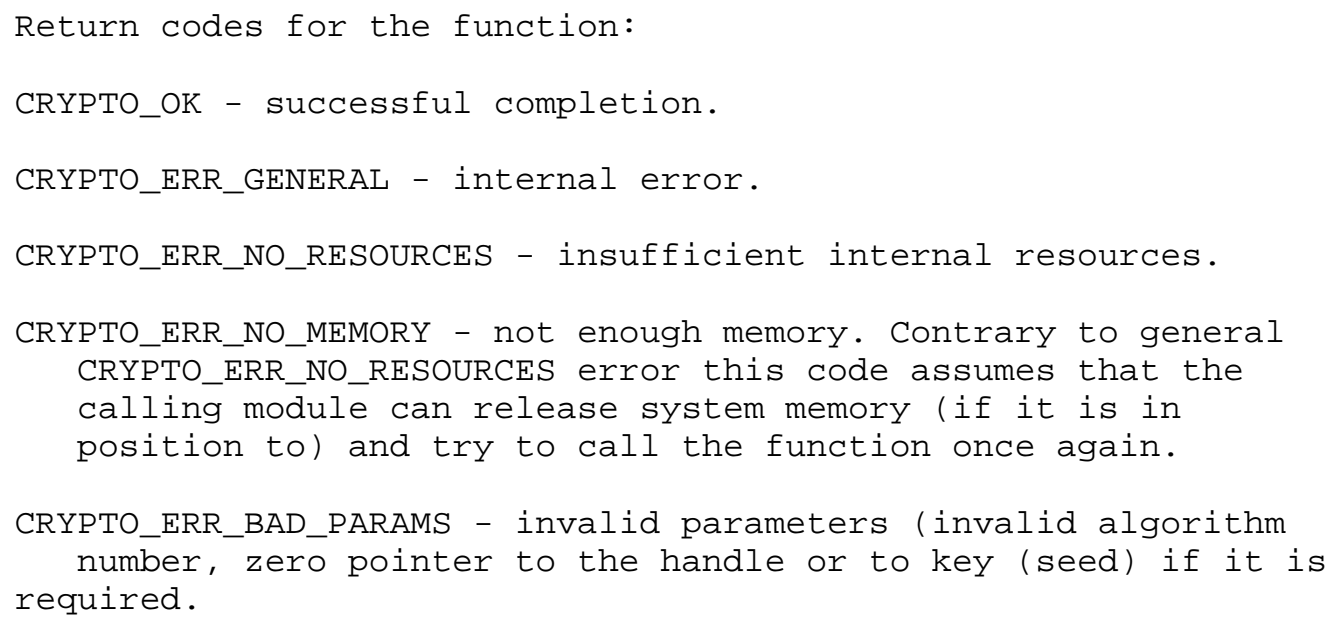

3.4. Cryptographic Context Reopening Function

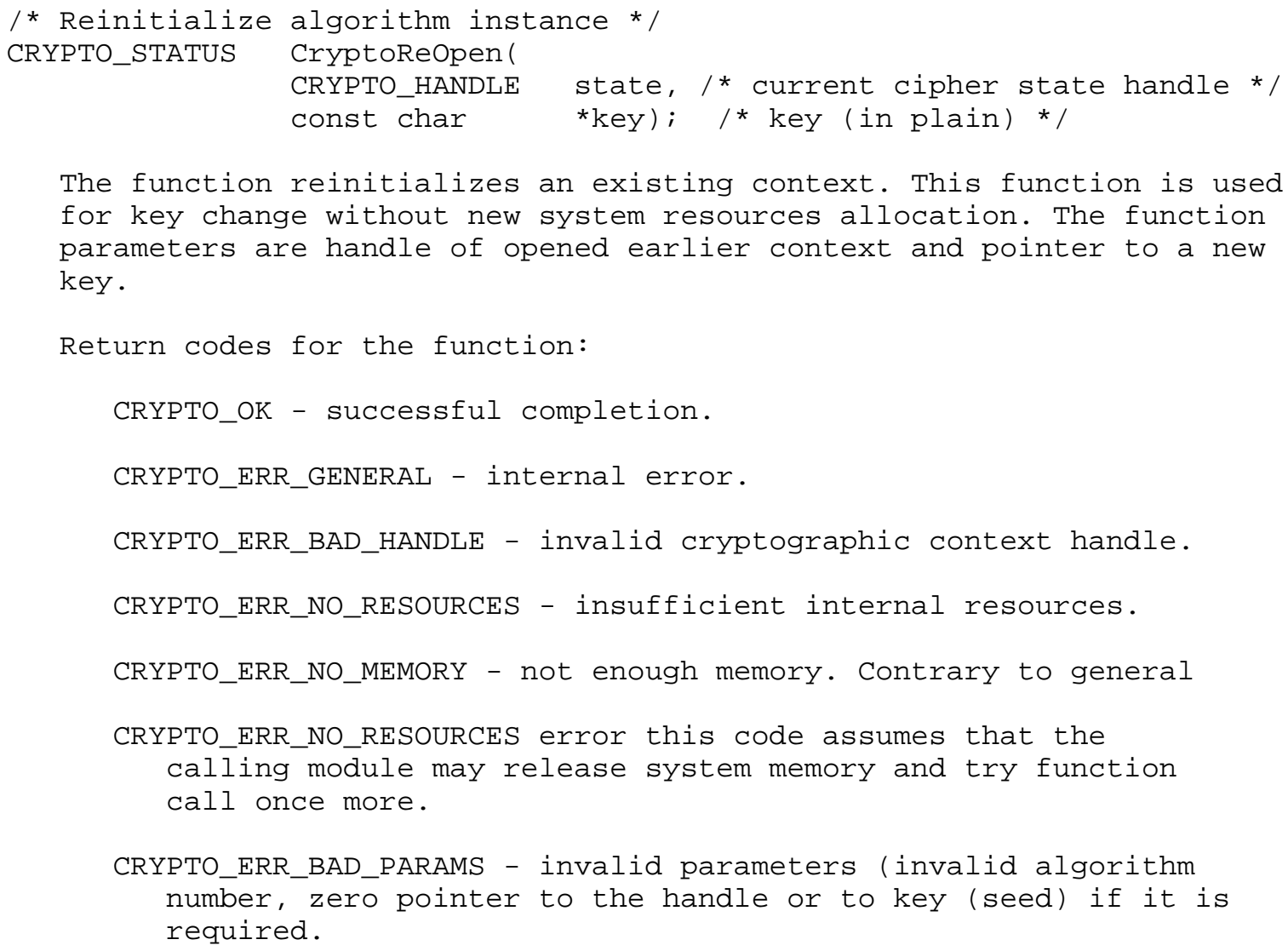




\subsection{Cryptographic Context Closing Function}

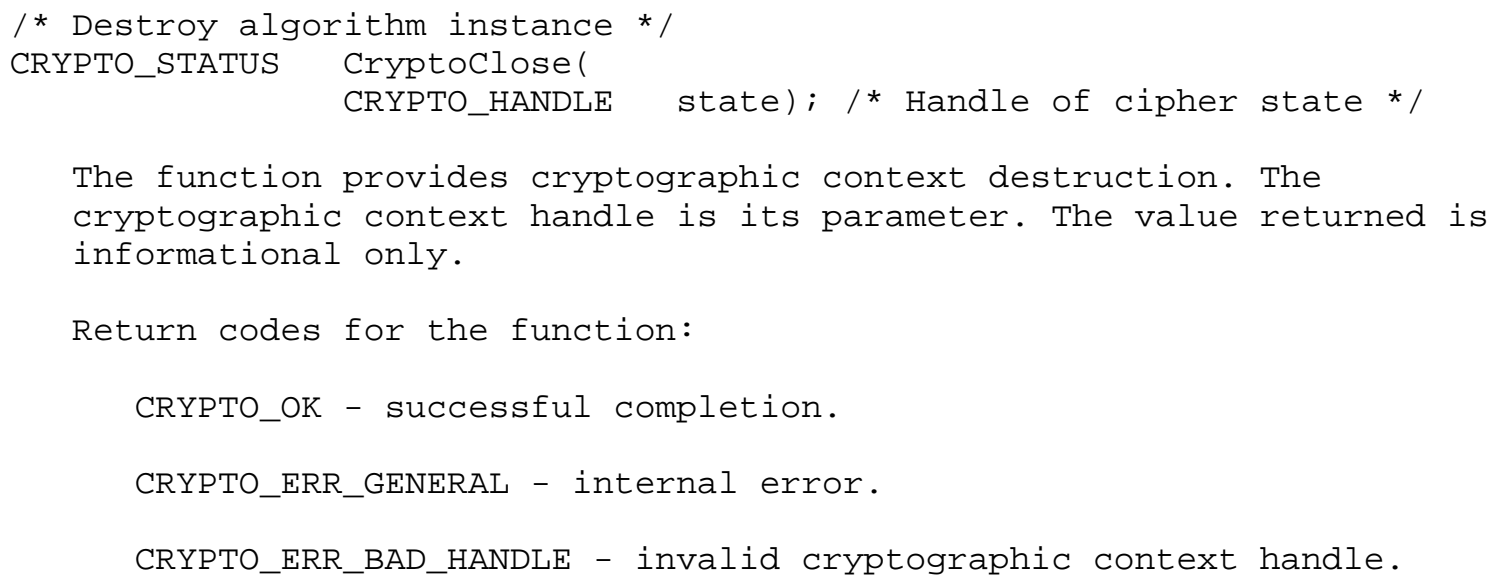

3.6. Key Verification Function

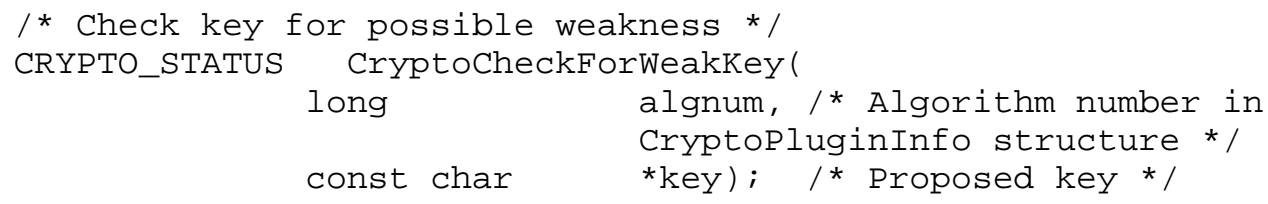




\subsection{Data Transformation Function}

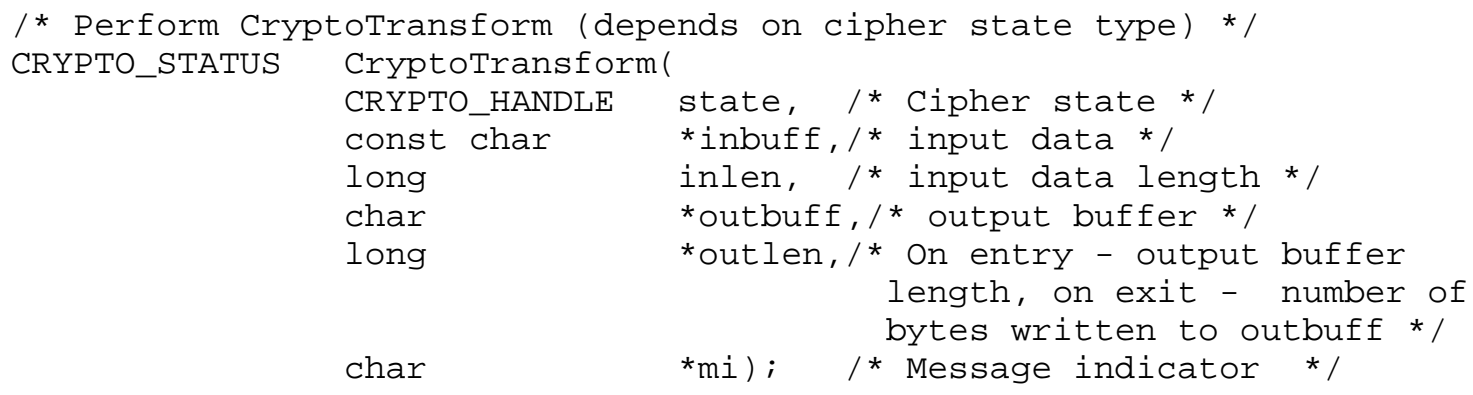

This is a cryptographic data transformation function. Function call results and function parameters are dependent on algorithm type. For algorithm types CRYTO_TYPE_ENCRYPT, CRYPTO_TYPE_DECRYPT, CRYPTO_TYPE_SIGN and CRYPTO_TYPE_VERIFY (items 3.7 .1 - 3.7.4)

function call results are history independent.

Note. Stream encryption algorithms may seem an "exception". However the same cryptoalgorithm handle must hide its history dependence. For algorithm types CRYPTO_TYPE_COMPRESS, CRYPTO_TYPE_UNCOMPRESS and CRYPTO_TYPE_HASH (items 3.7.5 - 3.7.7) function calls are history dependent. For the CRYPTO_TYPE_RANDOM algorithm function call may be for different implementations either dependent or independent on the history.

3.7.1. For CRYPTO_TYPE_ENCRYPT Algorithm Type:

The function encrypts input data. Its parameters are intended for:

inbuff - pointer to the input data. If this parameter is equal to NULL then the function should return the CRYPTO_ERR_BAD_PARAMS error code.

inlen - input data size (in bytes). If the size indicated in algorithm description is divisible by blocklen then padding is not carried out. Otherwise the algorithm either caries out padding according to the algorithm standard or returns appropriate error code (CRYPTO_ERR_BAD_PARAMS). The zero parameter is allowed so that the function quits at once and returns CRYPTO_OK code.

outbuff - output data buffer. NULL parameter value results in the outlen parameter setting to output buffer size required to encrypt the input buffer represented. In this case the CRYPTO_ERR_SMALL_BUFFER error should not be returned. 
outlen - Output buffer size is an input function parameter while the number of bytes written in the output buffer is the output parameter. Both the NULL parameter value and the zero value addressed result in CRYPTO_ERR_BAD_PARAMS code returned by the function.

mi - message indicator. Its content depends on whether the block or stream algorithm is applied. In the block algorithm case it is set to the last block encrypted. When the first block is encrypted mi parameter specifies initial initialization vector. In the stream algorithm case it is set to the offset of the first byte encrypted in the stream. If the algorithm uses the message indicator and the mi parameter value is set to NULL then function should return CRYPTO_ERR_BAD_PARAMS. If the algorithm (ECB Mode encrypting as an example) does not apply the message indicator then NULL value of $\mathrm{mi}$ is acceptable while non-NULL value should be ignored.

Returned values:

CRYPTO_OK - successful completion.

CRYPTO_ERR_GENERAL - internal error.

CRYPTO_ERR_BAD_HANDLE - invalid cryptographic context handle.

CRYPTO_ERR_NO_RESOURCES - insufficient internal resources.

CRYPTO_ERR_NO_MEMORY - not enough memory. Contrary to general CRYPTO_ERR_NO_RESOURCES error this code assumes that the calling module can release system memory (if it is in position tol and try to call the function once again.

CRYPTO_ERR_SMALL_BUFFER - insufficient output buffer size. CRYPTO_ERR_BAD_PARAMS - invalid parameters.

3.7.2. For CRYPTO_TYPE_DECRYPT Algorithm Type:

The function decrypts the input data. Its parameters are intended for:

inbuff - pointer to the input data. If the parameter is equal to NULL then the function should return the CRYPTO_ERR_BAD_PARAMS error code.

inlen - input data size (in bytes). When the parameter is set to zero the function quits at once and CRYPTO_OK code is returned. 
outbuff - output data buffer. NULL parameter value results in the outlen parameter setting to output buffer size required to decrypt the input buffer represented. In this case the CRYPTO_ERR_SMALL_BUFFER error should not be returned.

outlen - Output buffer size is an input function parameter while the number of bytes written in the output buffer is the output parameter. Both the NULL parameter value and the zero value addressed result in CRYPTO_ERR_BAD_PARAMS code returned by the function.

mi - message indicator. The content depends on whether the block or stream algorithm is applied. In the block algorithm case it is set to the last block encrypted. When the first block is decrypted mi specifies initial initialization vector. In the stream algorithm case it is set to the offset of the first byte decrypted in the stream. If the algorithm uses the message indicator and the mi parameter is set to NULL then function should return CRYPTO_ERR_BAD_PARAMS. If the algorithm (ECB Mode as an example) does not apply the message indicator then NULL value of $\mathrm{mi}$ is acceptable while non-NULL value should be ignored.

Returned values:

CRYPTO_OK - successful completion. CRYPTO_ERR_GENERAL - internal error.

CRYPTO_ERR_BAD_HANDLE - invalid cryptographic context handle. CRYPTO_ERR_NO_RESOURCES - insufficient internal resources.

CRYPTO_ERR_NO_MEMORY - not enough memory. Contrary to general CRYPTO_ERR_NO_RESOURCES error this code assumes that the calling module can release system memory (if it is in position to) and try to call the function once again.

CRYPTO_ERR_SMALL_BUFFER - insufficient output buffer size. CRYPTO_ERR_BAD_PARAMS - invalid parameters.

3.7.3. For CRYPTO_TYPE_SIGN Type Algorithm:

The function signs the input data. Its parameters are intended for: 


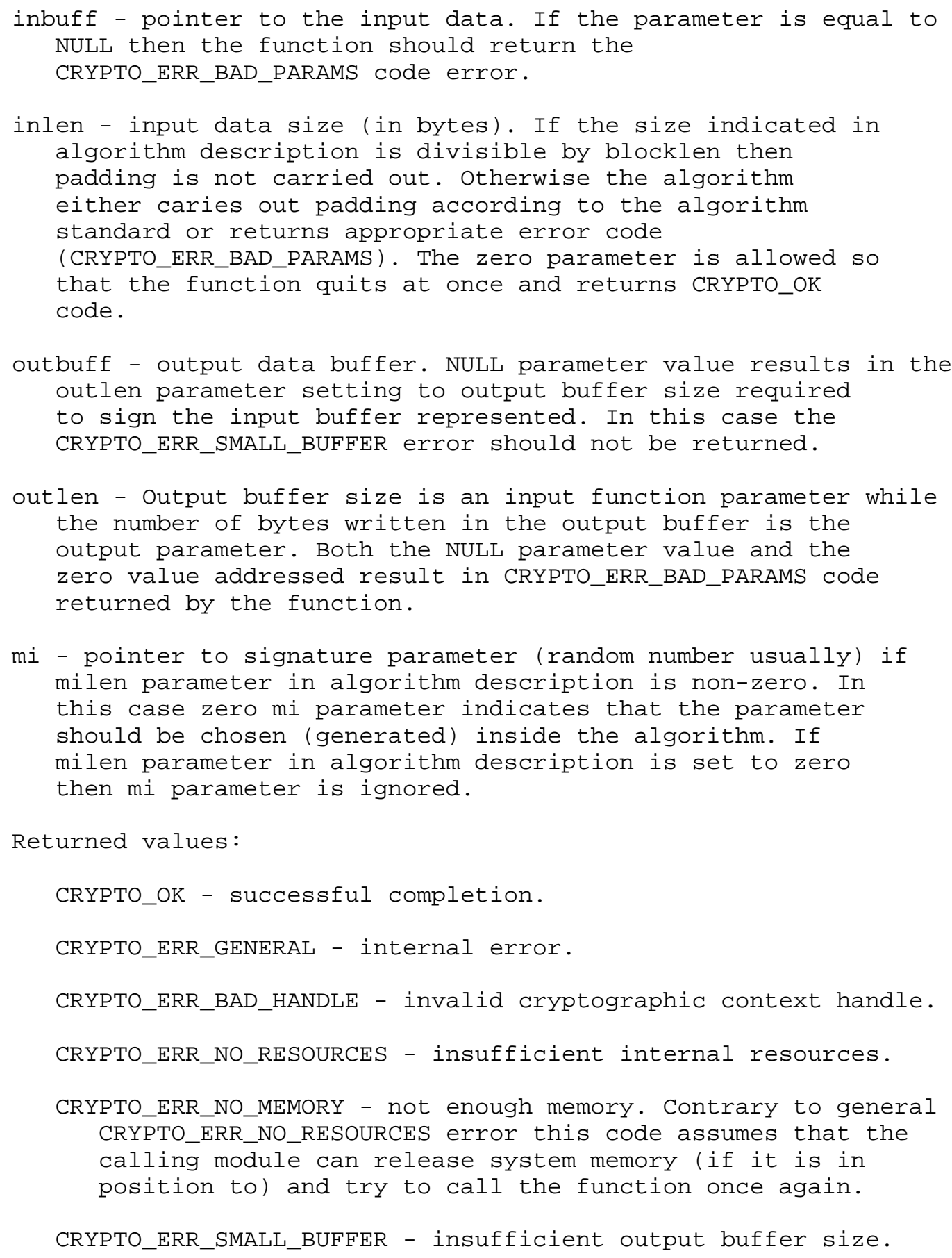




\section{CRYPTO_ERR_BAD_PARAMS - invalid parameters.}

3.7.4. FOr CRYPTO_TYPE_VERIFY Algorithm Type:

The function verifies input data signature. Its parameters are intended for:

inbuff - pointer to the input data. If the parameter is equal to NULL then the function should return the CRYPTO_ERR_BAD_PARAMS code error.

inlen - input data size (in bytes). The zero parameter is allowed so that the function quits at once and returns CRYPTO_OK code.

outbuff -pointer to the signature. If the parameter is set to NULL then the function returns CRYPTO_ERR_BAD_PARAMS error code. If the signature consists of several parts then they are combined to one array.

outlen - specifies the signature length if the signature length is set to zero in algorithm description structure. If non-zero value is specified in algorithm description structure then the parameter is ignored. If the signature consists of several parts then the maximum part length multiplied by the number of parts is specified.

mi - is not used.

Returned values:

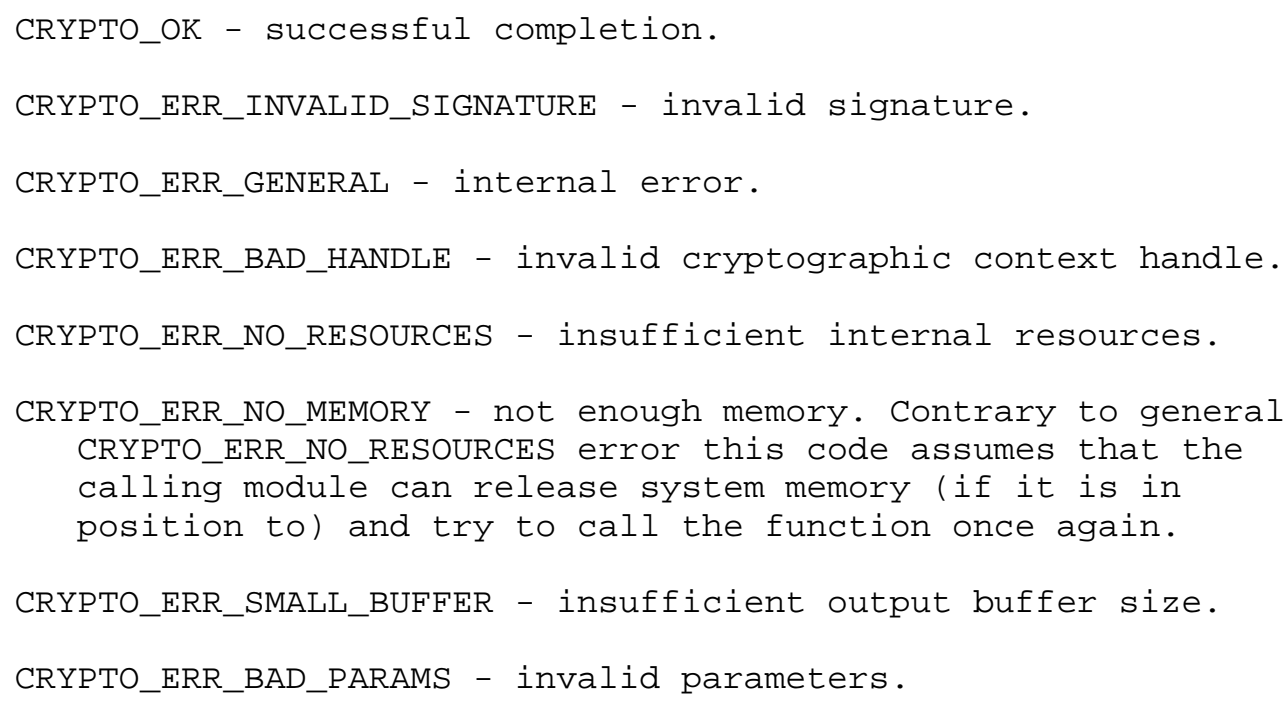




\subsubsection{FOr CRYPTO_TYPE_COMPRESS Algorithm Type:}

The function compresses the input data. Its parameters are intended for:

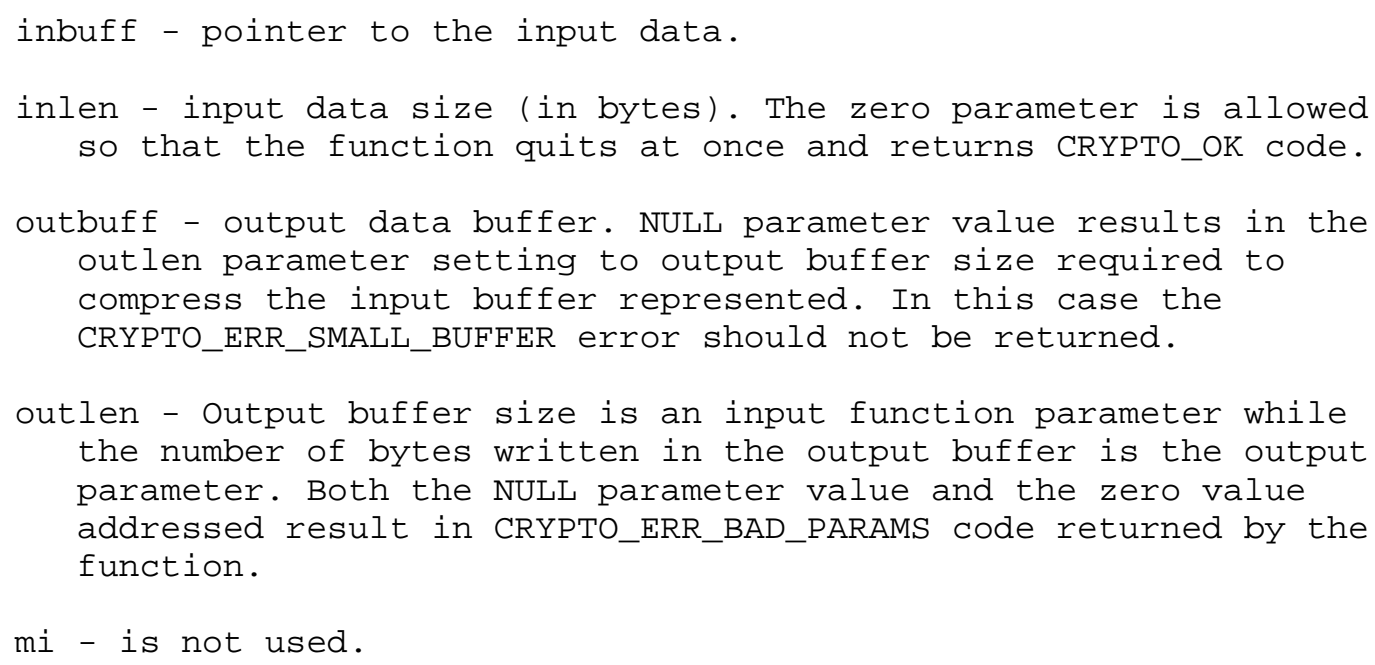


inlen - input data size (in bytes). The zero parameter is allowed so that the function quits at once and returns CRYPTO_OK code.

outbuff - output data buffer. NULL parameter value results in the outlen parameter setting to output buffer size required to decompress the input buffer represented. In this case the CRYPTO_ERR_SMALL_BUFFER error should not be returned.

outlen - Output buffer size is an input function parameter while the number of bytes written in the output buffer is the output parameter. Both the NULL parameter value and the zero value addressed result in CRYPTO_ERR_BAD_PARAMS code returned by the function.

mi - is not used.

Returned values:

CRYPTO_OK - successful completion.

CRYPTO_ERR_GENERAL - internal error.

CRYPTO_ERR_BAD_HANDLE - invalid cryptographic context handle.

CRYPTO_ERR_NO_RESOURCES - insufficient internal resources.

CRYPTO_ERR_NO_MEMORY - not enough memory. Contrary to general CRYPTO_ERR_NO_RESOURCES error this code assumes that the calling module can release system memory (if it is in position tol and try to call the function once again.

CRYPTO_ERR_SMALL_BUFFER - insufficient output buffer size. CRYPTO_ERR_BAD_PARAMS - invalid parameters.

3.7.7. For CRYPTO_TYPE_HASH Algorithm Type:

The function calculates the hash value of the input data. Its parameters are intended for:

inbuff - pointer to the input data. If the parameter is of NULL value then the function calculates cumulative hash value for the data represented (taking into account all previous data represented). If total length of all the data represented by the moment is divisible by blocklen and outbuff is non-NULL then it is returned to outbuff. Nothing is written in outbuff when the length is not divisible by blocklen. NULL inbuff indicates the last conversion when the input data is padded up 


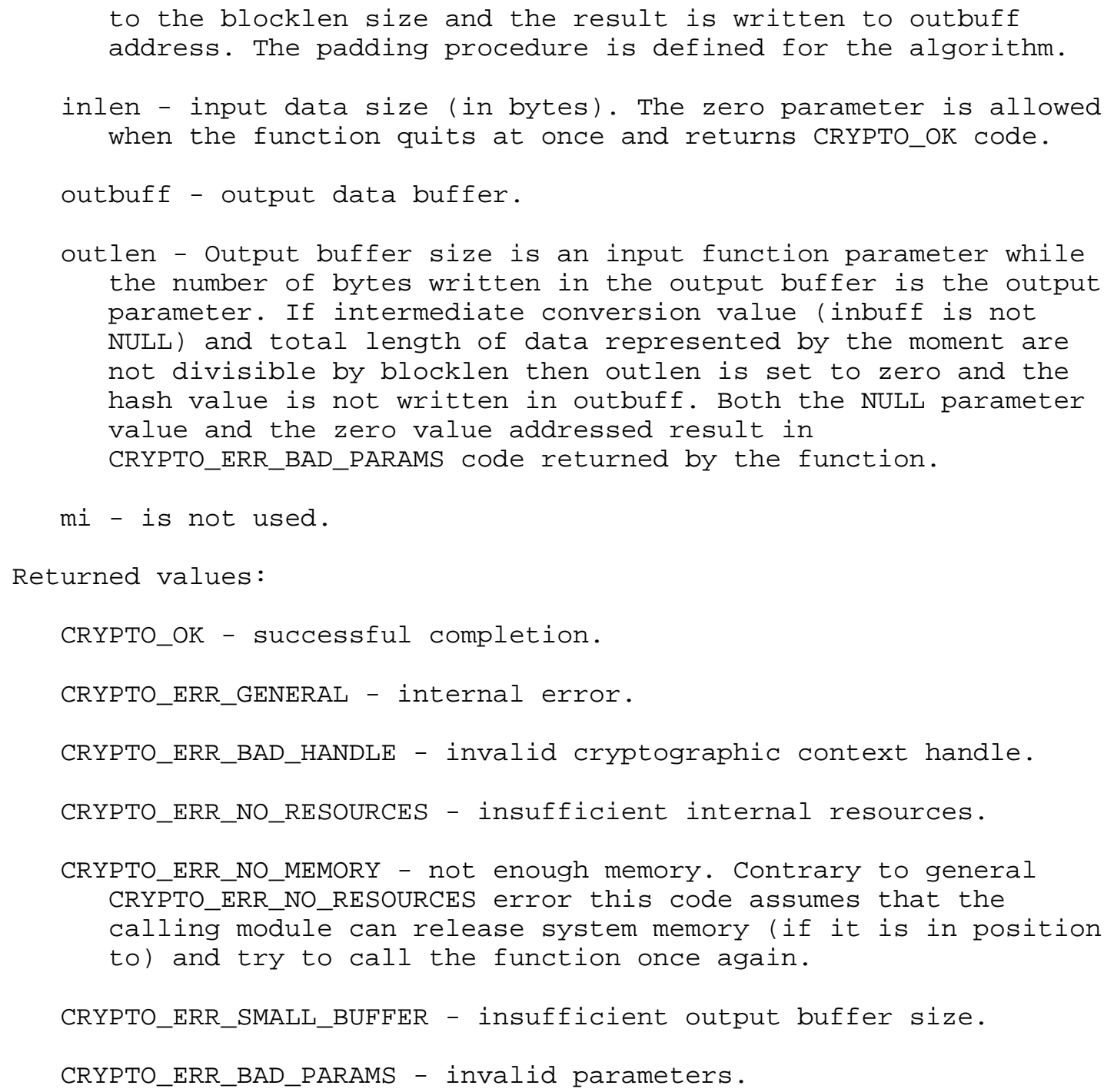




\subsubsection{FOr CRYPTO_TYPE_RANDOM Algorithm Type:}

The function generates a random number. Its parameters are intended for:

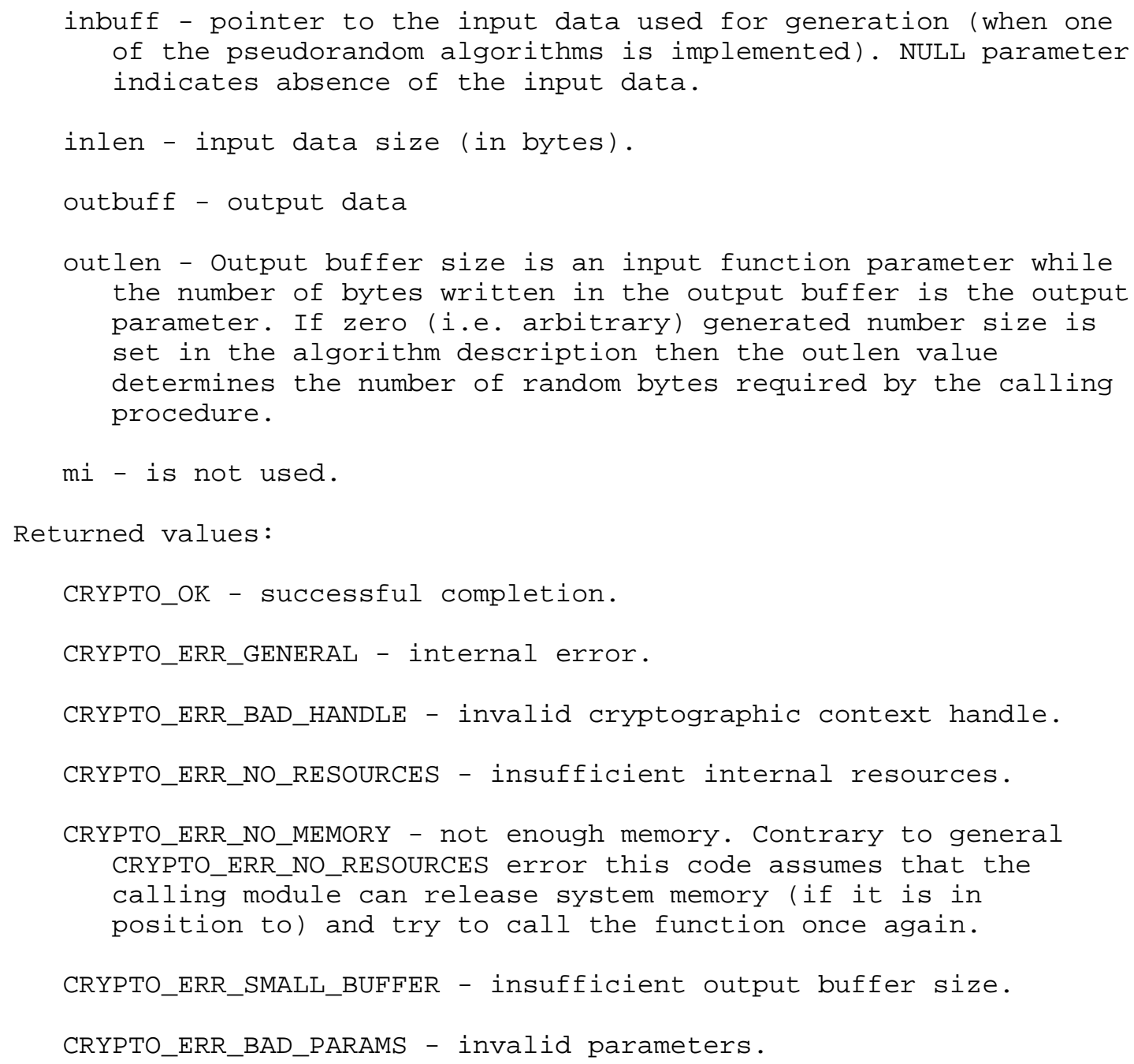




\subsection{Cryptographic Context Control Function}

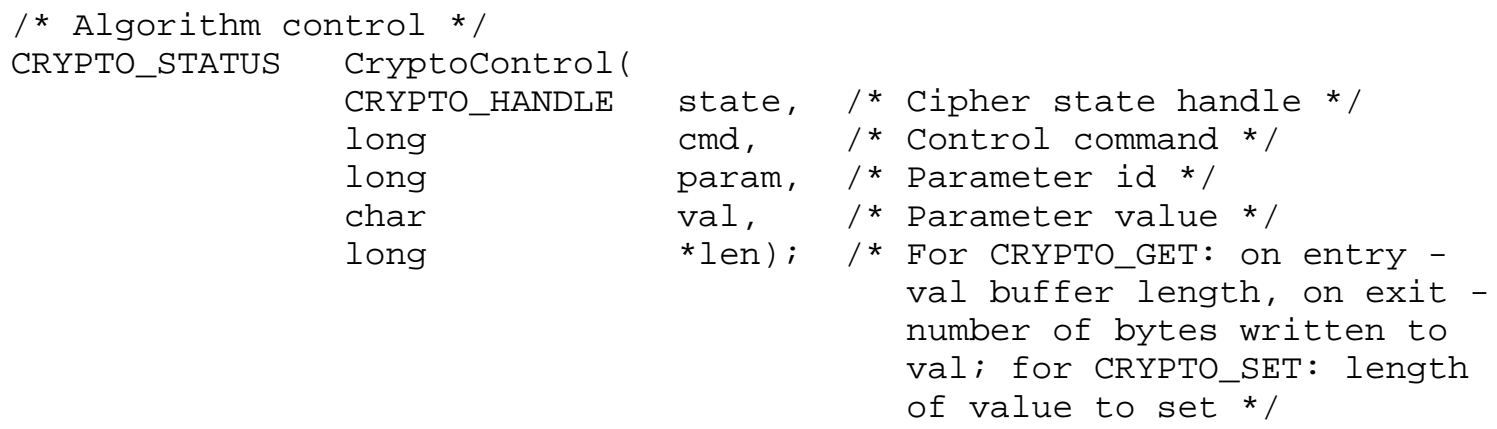

The function provides cryptographic context internal parameters management. It may be used to check context parameters or to change the context state, for example it may return information about cryptoalgorithm (is given context uses hardware encryption facilities), or it may "scroll" stream algorithms context if necessary, etc.

Description of parameters: 


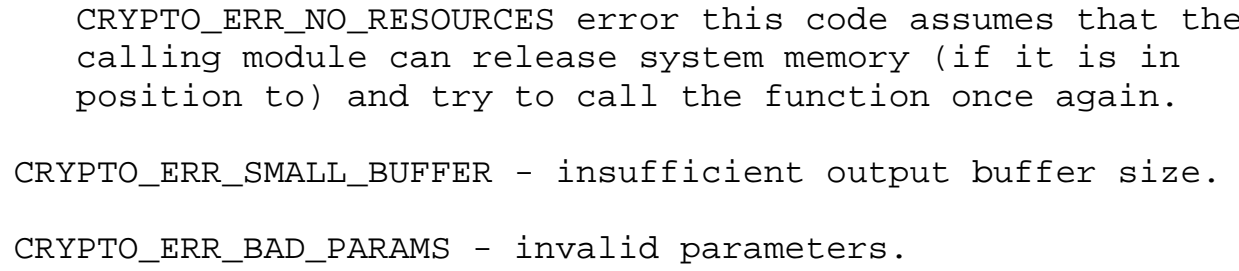

4. Cryptoplugin Registration Procedure

Cryptoplugin should be linked together with the cryptoplugin wrapper library delivered by the cryptoplugin's client developer according to the rules specified by the module-client developer for each platform. It should result in a driver (module) of appropriate operating system that implements the cryptolibrary functions. The driver should be one of the drivers loaded during operating system boot. The procedure of cryptoplugin driver installation should be defined, documented, and automated when necessary, by the cryptoplugin developer. At the beginning of operation the driver-client determines cryptoplugin driver availability and establishes interconnection with it. Both module-client configuration and current security policy determine data conversion algorithms to be chosen.

5. Security Considerations

Security issues are addressed throughout this memo.

6. References

[Schneier] Bruce Schneier, Applied Cryptography - Protocols, Algorithms, and Source Code in C (Second Edition), John Wiley \& Sons, Inc., 1996.

[IPsec] Kent, S. and R. Atkinson, "Security Architecture for the Internet Protocol", RFC 2401, November 1998.

[ISAKMP] Maughhan, D., Schertler, M. Schneider, M. and J. Turner, "Internet Security Association and Key Management Protocol (ISAKMP) ", RFC 2408, November 1998.

[IKE] Harkins, D. and D. Carrel, "The Internet Key Exchange (IKE)", RFC 2409, November 1998.

[TLS] Dierks, T. and C. Allen, "The TLS protocol Version 1.0", RFC 2246, January 1999. 
7. Author's Address

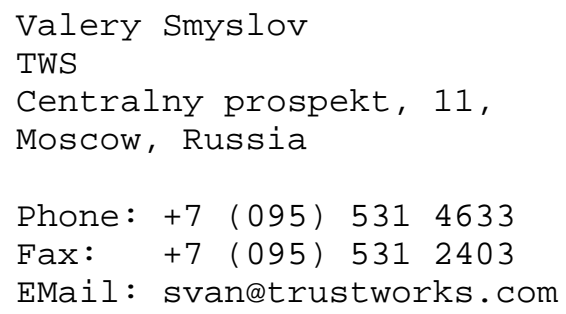


Appendix A. The interface specification as a C header file

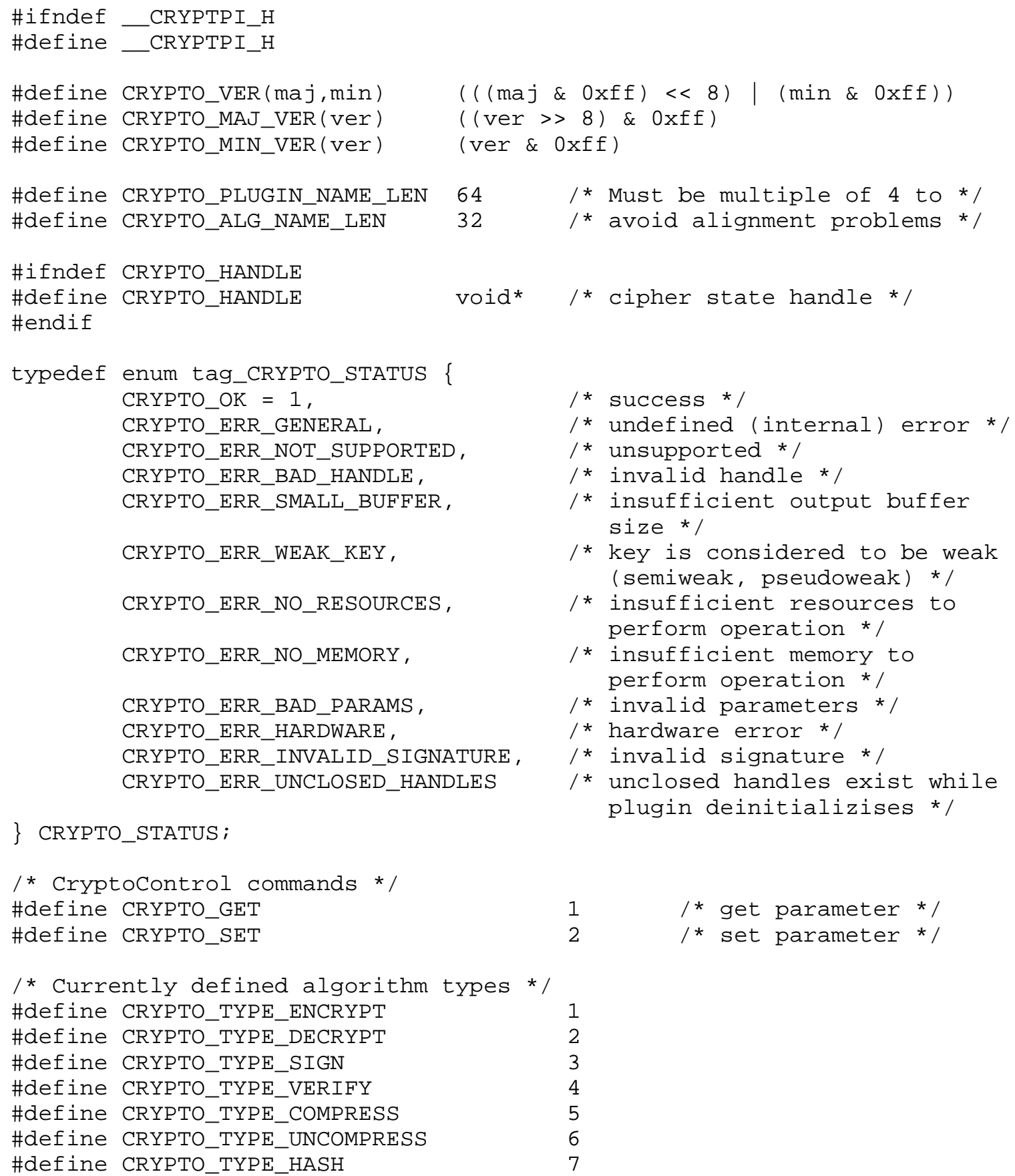




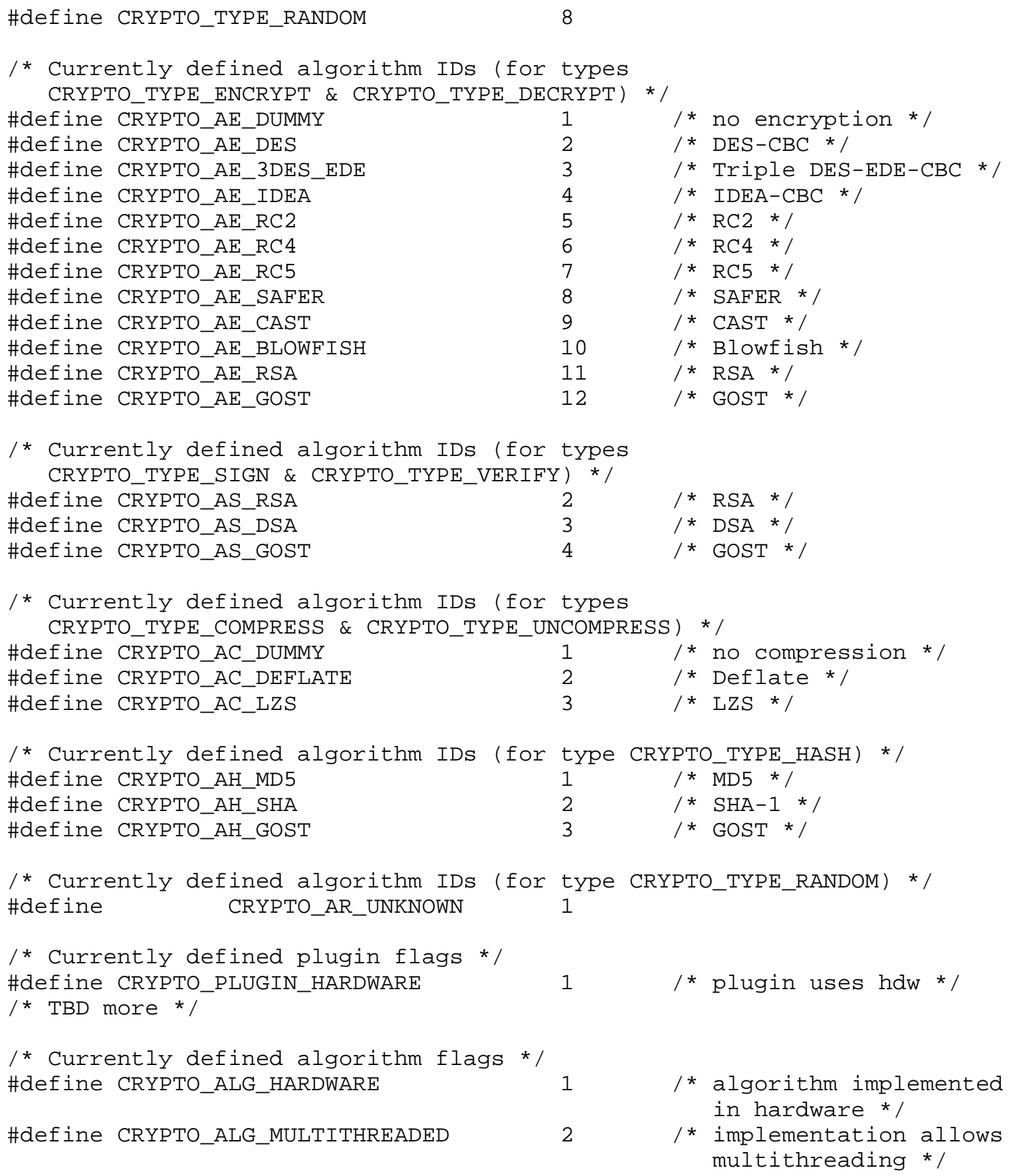




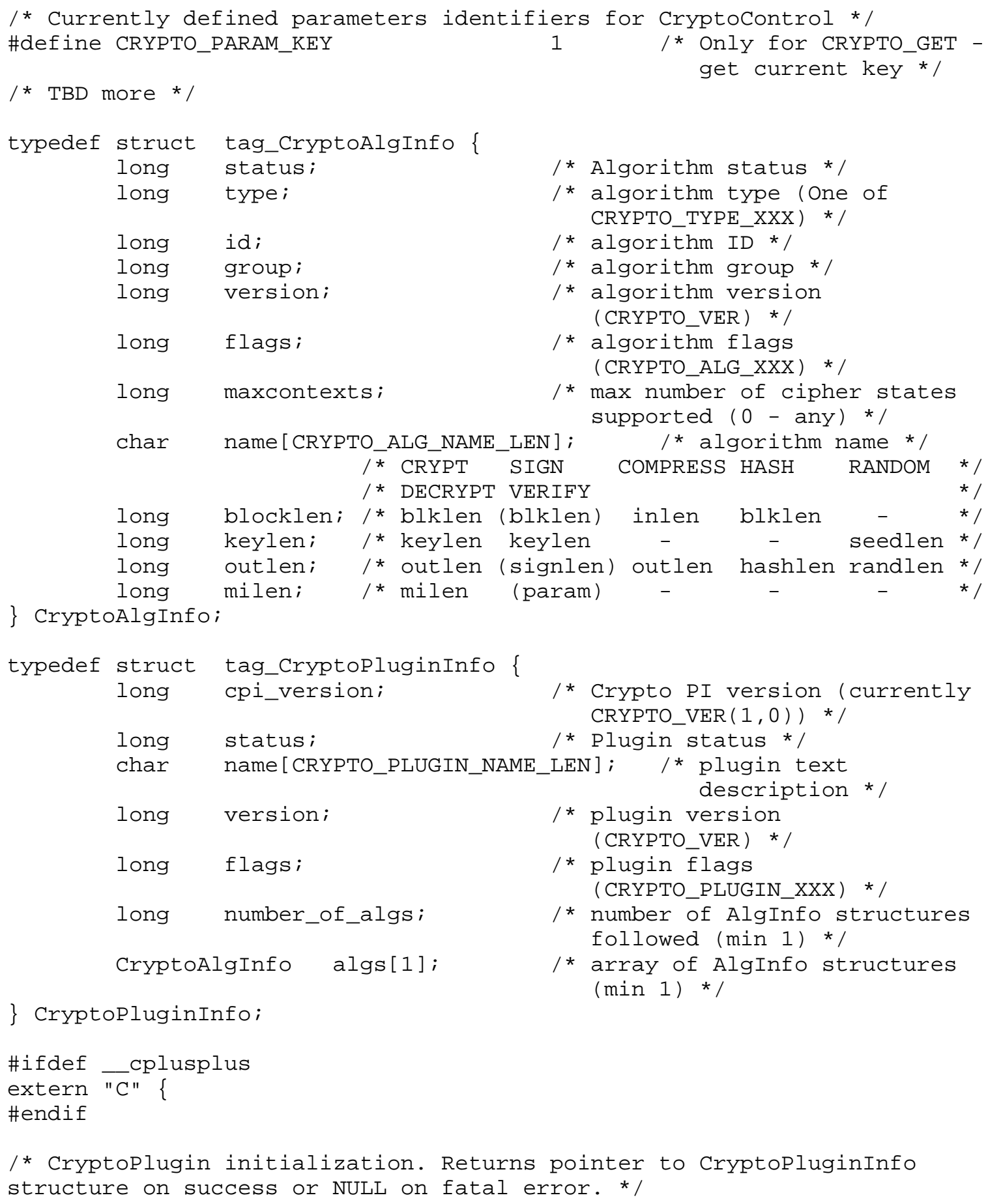




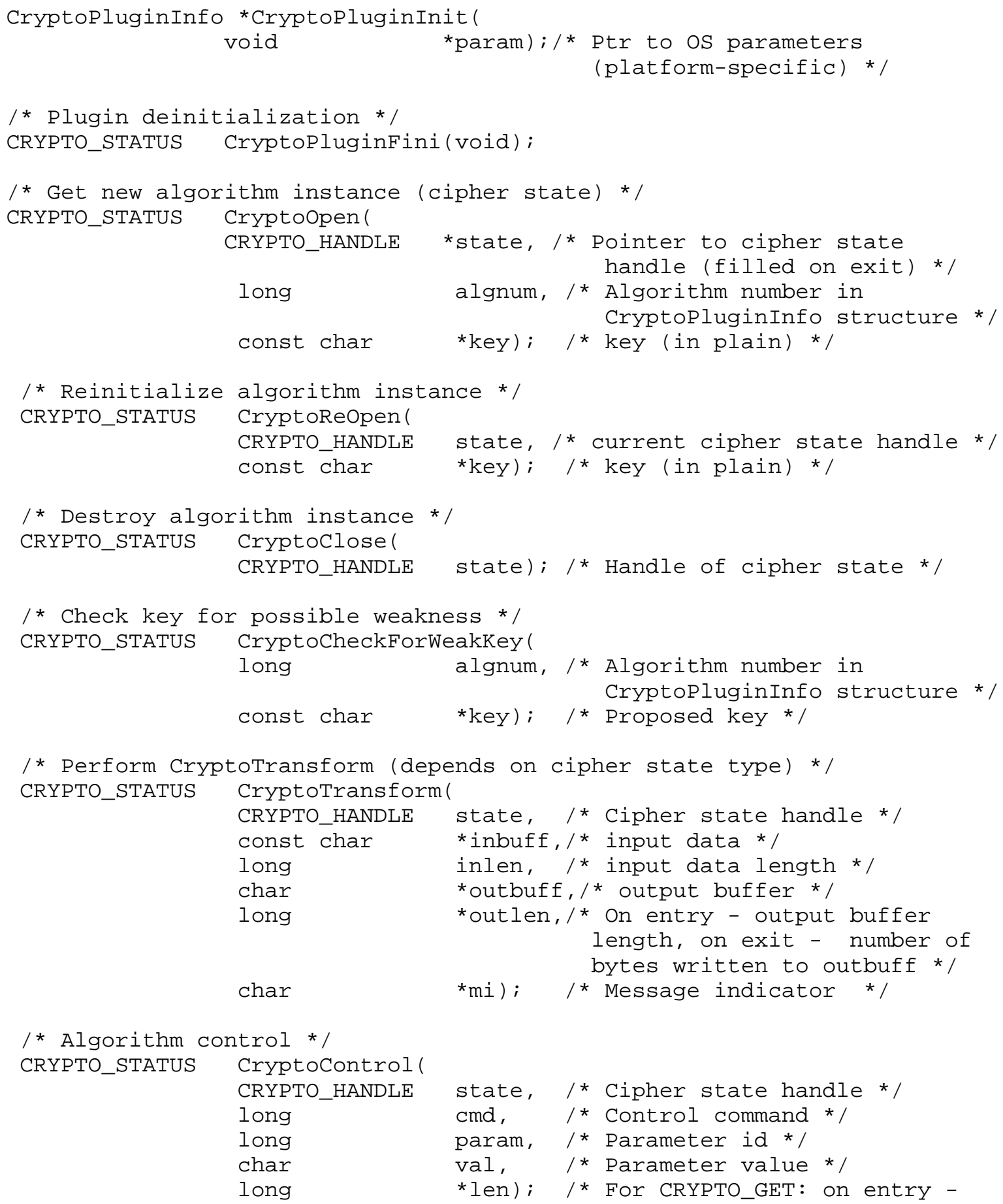


val buffer length, on exit number of bytes written to val; for CRYPTO_SET: length of value to set */

\#ifdef _cplusplus

\}

\#endif

\#endif / * CRYPTPI_H */ 
Full Copyright statement

Copyright (C) The Internet Society (1999). All Rights Reserved.

This document and translations of it may be copied and furnished to others, and derivative works that comment on or otherwise explain it or assist in its implementation may be prepared, copied, published and distributed, in whole or in part, without restriction of any kind, provided that the above copyright notice and this paragraph are included on all such copies and derivative works. However, this document itself may not be modified in any way, such as by removing the copyright notice or references to the Internet society or other Internet organizations, except as needed for the purpose of developing Internet standards in which case the procedures for copyrights defined in the Internet Standards process must be followed, or as required to translate it into languages other than English.

The limited permissions granted above are perpetual and will not be revoked by the Internet society or its successors or assigns.

This document and the information contained herein is provided on an "AS IS" basis and THE INTERNET SOCIETY AND THE INTERNET ENGINEERING TASK FORCE DISCLAIMS ALL WARRANTIES, EXPRESS OR IMPLIED, INCLUDING BUT NOT LIMITED TO ANY WARRANTY THAT THE USE OF THE INFORMATION HEREIN WILL NOT INFRINGE ANY RIGHTS OR ANY IMPLIED WARRANTIES OF MERCHANTABILITY OR FITNESS FOR A PARTICULAR PURPOSE.

Acknowledgement

Funding for the RFC Editor function is currently provided by the Internet society. 